\title{
Electroencephalographic abnormalities in sepsis patients in correlation to the calculated prognostic scores: A case series
}

\author{
Dimitrios Velissaris ${ }^{1 *}$, Nikolaos-Dimitrios Pantzaris ${ }^{1 *}$, Anastasia Skroumpelou ${ }^{2}$, \\ Panagiotis Polychronopoulos ${ }^{2}$, Vasilios Karamouzos ${ }^{3}$, Charalampos Pierrakos ${ }^{4}$, \\ Charalampos Gogos ${ }^{1}$, Menelaos Karanikolas ${ }^{5}$ \\ 'Internal Medicine Department, University Hospital of Patras, Greece; \\ ${ }^{2}$ Neurology Department, University Hospital of Patras, Greece; \\ ${ }^{3}$ Intensive Care Unit, University Hospital of Patras, Greece; \\ ${ }^{4}$ Intensive Care Department, Brugmann University Hospital, Brussels, Belgium; \\ ${ }^{5}$ Department of Anesthesiology, Washington University School of Medicine, St. Louis, MO, USA
}

* The two first authors of this article contributed equally and are listed as the first co-authors.

Address for Correspondence: Dr. Nikolaos-Dimitrios Pantzaris, MD, MEng, Register Doctor at Internal Medicine Department, University Hospital of Patras, Skoufa 62, Athens, 10680 Greece

E-mail: npantzaris@gmail.com

\begin{tabular}{|l|}
\hline Access this article online \\
\hline $\begin{array}{l}\text { Website: } \\
\text { www.intern-med.com }\end{array}$ \\
\hline $\begin{array}{l}\text { DOI: } \\
\text { 10.2478/jim-2018-0032 }\end{array}$ \\
\hline Quick Response Code: \\
\hline \\
\\
\\
\\
\end{tabular}

\section{ABSTRACT}

Objective: To evaluate the electroencephalographic (EEG) findings and correlate EEG findings with inflammatory biomarkers and the sepsis prognostic scores SOFA, SAPS II and APACHE II in patients who present in the Emergency Department with sepsis without clinical central nervous system involvement. Methods: The study included seventeen patients $(<70$ years old) with sepsis without central nervous system involvement presenting in the Emergency Department of the University Hospital of Patras, Greece. All patients underwent neurologic examination and EEG analysis on admission to the hospital and were treated according to the international guideline protocols for sepsis. Results: Six of seventeen sepsis patients had mild or moderate EEG abnormalities. We did not find any significant correlation between EEG abnormalities and inflammatory biomarkers (CRP, WBC) or commonly used prognostic sepsis scores. Conclusions: EEG could serve as a useful tool to identify brain alterations at an early stage in sepsis, before clinical sings of encephalopathy can be detected. However, the presence of EEG abnormalities does not correlate with sepsis severity as measured by the commonly used prognostic sepsis scores SOFA, APACHE II or SAPS II. Because this was a small single center observational study, large multi-center studies are warranted to confirm these findings.

Key words: sepsis, electroencephalogram, EEG, sepsis severity scores, encephalopathy, SAE

\section{INTRODUCTION}

Brain dysfunction is a frequent complication of sepsis even in cases of extra-cranial origin and is related to several underlying mechanisms. Encephalography (EEG) seems to be a useful tool in detecting the presence of encephalopathy in patients with sepsis. Although EEG is not a specific test, it is sensitive and can detect abnormalities even when clinical neurologic examination is normal. The aim of this study was to document the EEG abnormalities and search for correlations between EEG findings and commonly used severity and prognostic scores (SOFA, APACHE II, SAPS II) in patients who presented in the emergency department (ED) with sepsis but without clinical signs of encephalopathy.

\section{MATERIAL AND METHODS}

\section{Study population and design}

This prospective observational study included 17 patients with sepsis who presented in the Emergency Department of the University Hospital of Patras, Greece over a period of 6 months in winter of 2015 and spring of 2016. The diagnosis of sepsis was established according to the current international criteria, defined as life threatening organ dysfunction with SOFA score $\geq 2 .^{[1]}$ Exclusion criteria were: 1) central nervous system (CNS) infection, 2) abnormal findings in neurologic 
examination, 3) age over 70 years and 4) presence of systemic disease that could affect the CNS. Patients with metabolic alterations (dysglycaemia, plasma sodium abnormalities, renal and hepatic failure) or drug toxicity that could affect the CNS were also excluded. The study protocol was approved by the Research and Ethics Committee of the University Hospital of Patras, Greece. Consent for participation in the study was obtained from each patient or a designated family member and was documented in writing. The research team included Internal medicine and Neurology Consultants, resident physicians, staff nurses and medical students.

\section{Data collection}

Each patient was initially assessed by a Neurology and Internal Medicine Consultant. Blood samples were drawn from each patient and the analysis included complete blood count, a full biochemistry panel and arterial blood gas analysis. The APACHE II, SAPS II and SOFA scores were calculated for each patient on admission in the Emergency Department. Neurologic assessment included detailed clinical examination and electroencephalogram (EEG) analysis within 6 hours of admission. EEG was recorded in all patients using a Galileo NT clinical EEG system (EB Neuro, Italy), with 21 channels and international 10/20 electrode placement and EEG signals were acquired using standard parameters [Sensitivity $10 \mu \mathrm{V} / \mathrm{mm}$, Low Frequency Filter (LFF) $0.500 \mathrm{~Hz}$ and High Frequency Filter (HFF) $70 \mathrm{~Hz}$ ]. EEG studies were conducted in a quiet room, with participants instructed to stay awake but keep eyes closed, and EEG recording lasted for 20-25 minutes. EEG findings were characterized as abnormal when any excessive theta waves, predominant delta waves, tri-phasic waves, suppression or burst-suppression patterns or any combination of the above was documented.

\section{Statistical analysis}

Data normality was assessed using the Shapiro-Wilk test, and the data were summarized using descriptive statistical tools including frequencies for discrete variables and mean \pm standard deviation or median (range) for continuous variables as appropriate. Independent sample t-tests or ANOVA were used to compare the means between groups as appropriate and Scheffe's test was used for post-hoc comparisons. Correlation between age, prognostic scores and EEG findings was assessed using the Pearson's $r$ or Spearman's rho coefficient as appropriate, and $P<0.05$ was considered significant for all the comparisons. All data analysis was conducted using the IBM SPSS Statistics version 23.0 software package.

\section{RESULTS}

\section{Demographics and clinical severity data}

Minimum patient age was 15 years and maximum age was 70 years (mean 43.35 years, SD 17.41 years). 9 patients $(52.9 \%)$ were male and $8(47.1 \%)$ were female. The source of infection was the chest in $5(29.4 \%)$ patients, the urinary tract in $6(35.3 \%)$, the abdominal cavity in $3(17.6 \%)$, and soft tissue in $3(17.6 \%)$ cases. EEG was normal in 11 patients and abnormal in 6. Age had highly significant positive correlation with SOFA score (Spearmans's rho $0.561, P=0.019$ ), with APACHE II score (Pearson's $r=0.596, P=0.012$ ) and with SAPS III score (Pearson's $r=0.715, P=0.001)$. The positive correlation of the variable "age" with the APACHE II and with the SAPS III scores was expected because age is one of the variables used to calculate these scores. Patient data and prognostic scores calculated in the Emergency Department are presented in Table 1.

\begin{tabular}{|c|c|c|c|c|c|c|c|}
\hline Patient ID & Age & Gender & Source of sepsis & EEG & SOFA & APACHE II & SAPS II \\
\hline 1 & 53 & female & Urinary tract & 1 & 6 & 13 & 39 \\
\hline 2 & 68 & male & Soft tissue & 1 & 4 & 13 & 35 \\
\hline 3 & 36 & female & Urinary tract & 1 & 2 & 6 & 17 \\
\hline 4 & 23 & female & Urinary tract & 1 & 2 & 6 & 18 \\
\hline 5 & 30 & female & Urinary tract & 1 & 2 & 3 & 11 \\
\hline 6 & 40 & male & Chest & 1 & 5 & 13 & 35 \\
\hline 7 & 62 & female & Chest & 3 & 3 & 13 & 38 \\
\hline 8 & 70 & male & Urinary tract & 3 & 3 & 12 & 29 \\
\hline 9 & 15 & male & Gl tract & 2 & 2 & 5 & 17 \\
\hline 10 & 65 & male & Chest & 1 & 3 & 21 & 36 \\
\hline 11 & 52 & male & Chest & 1 & 4 & 14 & 30 \\
\hline 12 & 38 & female & Urinary tract & 2 & 4 & 5 & 20 \\
\hline 13 & 50 & male & Soft tissue & 1 & 2 & 6 & 13 \\
\hline 14 & 51 & female & GI tract & 1 & 4 & 9 & 26 \\
\hline 15 & 40 & male & Chest & 1 & 2 & 4 & 16 \\
\hline 16 & 26 & male & Gl tract & 2 & 3 & 15 & 11 \\
\hline 17 & 18 & female & Soft tissue & 2 & 2 & 7 & 19 \\
\hline
\end{tabular}

EEG: $1=$ normal, 2 = Mild non-specific abnormalities, $3=$ Moderate non-specific abnormalities. Gl: Gastrointestinal. 
Clinical neurological findings

All patients had a Glasgow Coma Scale (GCS) of 15, detailed neurologic examination did not detect any neurologic deficits in any patient and mental and cognitive status were reported as normal in all patients.

\section{EEG findings}

Findings from the EEG recordings of the 17 patients were the following: Eleven patients had normal EEG and 6 patients showed non-specific EEG disturbances. Four of the 6 patients had mild non-specific EEG disturbances (mild slowing in the theta range), whereas 2 patients had moderate non-specific EEG disturbances (intermittent delta activity on a mildly slow background). Triphasic waves or burst-suppression were not identified in any patient. Observed EEG abnormalities were classified based on the classification proposed by Young, as Class 1 in 11 of the patients, Class 2 in 4 patients and Class 3 in 2 patients. ${ }^{[2]}$

Correlation of EEG findings with prognostic scores Abnormal EEG findings did not show any significant correlations with the serum CRP levels or WBC, and there was no significant correlation between the EEG findings and SOFA, APACHE II, or SAPS III sepsis severity scores (Table 2). However, age was different between patients with normal EEG (Median 50, minimum 23, maximum 68) versus patients with Class 1 abnormalities (Median 22, minimum 15, maximum 38) or Class 2 EEG abnormalities (Median 66, minimum 62, maximum 70), and this difference was highly significant $(P=0.005)$ (Figure 1). Scheffe post-hoc testing showed that the patients with Class 1 abnormalities had a significantly lower age compared to the patients with normal EEG $(P=0.034)$ and also compared to the patients with Class 2 abnormalities $(P=0.007)$.

\section{DISCUSSION}

Sepsis-associated encephalopathy is the term used to define brain dysfunction related to infections that are principally located outside the central nervous system. This syndrome has also been referred to as sepsis-associated brain dysfunction (SABD) $)^{[3-4]}$ and may ensue before signs of organ failure appear, ${ }^{[5]}$ with incidence ranging from $9 \%$ to $71 \%$ and clinical manifestations ranging from confusion to coma. Decreased brain perfusion is considered as a major determinant in the pathogenesis of brain dysfunction in sepsis, but various other mechanisms can be involved but vary from patient to patient. ${ }^{[6]}$ These mechanisms include inflammatory and non-inflammatory processes affecting the endothelial cells, glial cells and neurons resulting in blood-brain barrier breakdown, and derangements of intracellular metabolism, which can lead to cell death. ${ }^{[7}$ Furthermore, systemic consequences of sepsis, such as prolonged inflammation, severe hypoxemia, and persistent hyperglycemia may contribute to aggravation of sepsisinduced brain dysfunction or injury. ${ }^{[8]}$ Morbidity and mortality are directly related to its severity. Sepsis-related encephalopathy consists of acute changes in mental status, alterations of sleep/wake cycle, cognition, disorientation, impaired attention and/or disorganized thinking. In more severe cases agitation and hallucinations can be observed. Less frequent symptoms are rigidity, asterixis, tremor and multifocal myoclonus. ${ }^{[8-9]}$ Delirium and mild EEG slowing seem to be the earliest features of sepsis-related encephalopathy. ${ }^{[10-11]}$ Diagnostic tools useful for detection of brain dysfunction are the electroencephalogram, brain MRI and somatosensory evoked potentials, but blood tests, such as plasma S-100b protein and neuron-specific enolase levels can also be helpful. ${ }^{[7]} \mathrm{EEG}$ is almost sensitive but not specific for SABD. CT of the brain and brain

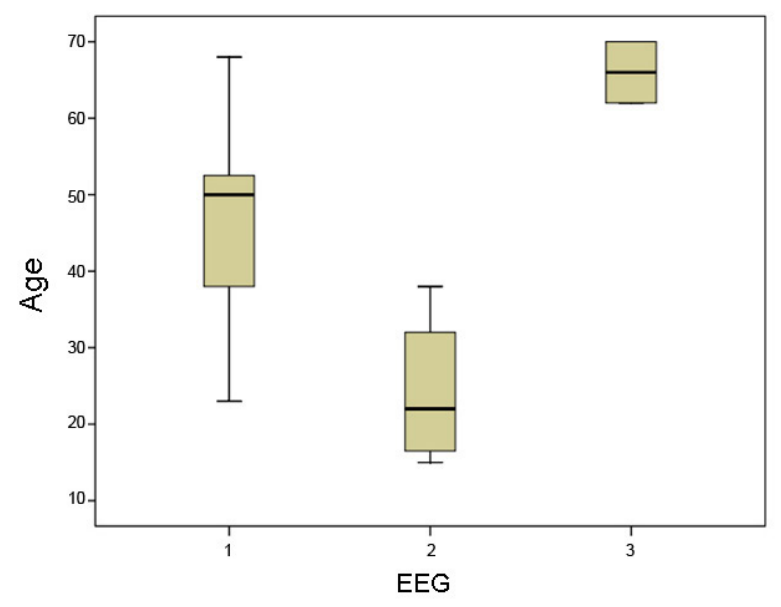

Figure 1: Age distribution in patients with normal EEG (1) vs. patients with mild EEG abnormalities (2) vs. patients with moderate EEG abnormalities (3). The overall difference between the three EEG groups is highly significant $(P=0.005)$.

\begin{tabular}{lll}
\hline \multicolumn{2}{l}{ Table 2: Bivariate correlation between EEG and other variables of interest Spearman's rho coefficient analysis } \\
\hline Variable of interest & Spearman's Rho & P value \\
\hline Age & -0.083 & 0.753 \\
Serum CRP & 0.069 & 0.791 \\
WBC & 0.074 & 0.778 \\
APACHE II & 0.028 & 0.914 \\
SAPS II & 0.013 & 0.960 \\
SOFA & -0.082 & 0.754 \\
\hline
\end{tabular}

No significant correlations identified. 
MRI generally do not reveal specific abnormalities, while Somatosensitive Evoked Potentials are sensitive markers of sepsis-associated cerebral dysfunction. ${ }^{[12]}$

Electroencephalography measures spontaneous electrical activity generated by synaptic transmission in the superficial layers of the cerebral cortex and modulated by subcortical structures from the upper brainstem to the thalamus. The raw EEG can be assessed visually or analyzed by quantitative methods that extract descriptive features like frequency, power, linearity and amplitude. EEG tracing and power is correlated with the cerebral blood flow, ${ }^{[13]}$ a fact that reveals its utility in conditions with altered brain perfusion such as sepsis. The EEG is fairly sensitive and can show abnormalities even in cases that neurological examination of the sepsis patient is normal. Sepsis can be associated with electrographic seizures or periodic epileptiform discharges, increased theta rhythms, triphasic waves and less often burst suppression patterns. ${ }^{[1,14-15]}$ EEG findings are related to severity of the encephalopathy: mild cases only have EEG slowing in the theta range, whereas EEG in more severe cases shows intermittent rhythmic delta activity, followed by more persistent delta activity as the predominant rhythm. Triphasic waves may also occur, and finally, burst-suppression ensues in severe cases. A classification by Young et al. describes 5 stages of progressively worsened EEG pattern related to worsened outcome: 1 $=$ refers to normal EEG, 2 = excessive theta waves, $3=$ predominant delta waves, $4=$ triphasic waves and $5=$ burst suppression, in ascending order of severity. ${ }^{[2]}$

In our case-series, 6 out of 17 sepsis patients had EEG abnormalities at the time of presentation to the ED, in the absence of other causes that could justify these findings. Therefore, it is important to remind clinicians that sepsis can affect the brain before the onset of clinical neurologic deterioration. Although this study had a small sample size, the finding that $35 \%$ of patients had EEG abnormalities is still important. A systematic review by Hosokawa et al. based on the 1976 citations evaluated the role of EEG and Evoked Potentials (EPs) in sepsis patients and showed that EEG and EPs abnormalities are present in most patients: the incidence of EEG abnormalities in sepsis ranged from $12 \%$ to $100 \%$ for background abnormality and from $6 \%$ to $12 \%$ for the presence of triphasic waves. ${ }^{[16]}$ However, in contrast to the patient populations in the previous review, our study is different because it evaluated the non-ICU sepsis patients without clinical encephalopathy. All patients in our study were admitted from the community and treated in the Internal Medicine Department. EEG was performed within 6 hours of admission and at that time there were no signs of septic shock and no need for administration of vasoactive agents.
Many studies have reported the presence of EEG abnormalities alone or in combination to other tests such as evoked potentials (EPs) during sepsis. However, the role of these tests in the diagnosis and prognostic assessment of sepsis-related encephalopathy is unclear. Despite intensive research in sepsis, we still do not know at what stage of sepsis the first signs of encephalopathy appear. ${ }^{[17]}$ Alterations of brain electrical activity are markers of sepsisinduced brain injury and there is evidence supporting the importance of EEG in the detection and prognostication of sepsis-related encephalopathy. However, indication for EEG is not clearly established and further research based on large trials with more homogenous sepsis population are needed. Clinicians should be aware of the possible effects of sepsis on the brain even when detailed negative neurologic examination is unremarkable. Because the assessment of brain function with EEG is a safe, lowcost and easy to perform method that may identify brain alterations early, intensive brain function monitoring can be a reasonable option in certain cases.

Finally, another important finding from this case series, is the absence of correlation between the EEG findings and the calculated prognostic scores of sepsis upon admission. All patients did well, did not require ICU admission, were admitted and treated in the Internal Medicine Department and eventually were discharged home. Studies show that mortality rates are increased up to $67 \%$ in cases where EEG patterns show burst suppressions, electroencephalographic seizures and periodic discharges compared to good outcomes when EEG is normal. ${ }^{[15,18]}$ In this case series, 6 out of 17 sepsis patients had EEG abnormalities on admission but finally did well. Sepsisrelated encephalopathy is a multifaceted syndrome with complex mechanisms and the underlying pathology is not completely understood. It can occur early during sepsis and usually precedes sepsis-related multiple organ failure. Although the sample size of this study is small, we believe this case series adds useful information to the literature regarding the presence of brain involvement as evidenced by EEG abnormalities in the early stage of sepsis. Our findings suggest that EEG seems to be more sensitive than clinical criteria for encephalopathy and therefore could be a useful complement to clinical assessment in order to define the severity of disease in patients with sepsis.

\section{CONCLUSIONS}

Early assessment of EEG could be a useful tool in the initial evaluation of patients with sepsis. Clinicians should be aware that brain dysfunction evidenced by EEG abnormalities can be present early in sepsis, in the absence of obvious clinical encephalopathy. In this case series, the presence of EEG abnormalities did not correlate with 
the widely used sepsis severity scores SOFA, APACHE II and SAPS III, and we believe this finding deserves further assessment in future larger trials. The role of EEG as a first line non-invasive tool in the assessment and management of encephalopathy in sepsis deserves further investigation.

\section{Conflict of Interest}

This work was supported solely by the Department funds. All authors state that they do not have any conflicts of interest to report.

\section{REFERENCES}

1. Singer M, Deutschman CS, Seymour CW, Shankar-Hari M, Annane D, Bauer M, et al. The Third International Consensus Definitions for Sepsis and Septic Shock (Sepsis-3). JAMA 2016; 315: 801-10.

2. Young GB. Encephalopathy of infection and systemic inflammation. J Clin Neurophysiol 2013; 30: 454-61.

3. Gofton TE, Young GB. Sepsis-associated encephalopathy. Nat Rev Neurol 2012; 8: 557-66.

4. Adam N, Kandelman S, Mantz J, Chretien F, Sharshar T. Sepsis-induced brain dysfunction. Expert Rev Anti Infect Ther 2013; 11: 211-21.

5. Consales G, De Gaudio AR. Sepsis associated encephalopathy. Minerva Anestesiol 2005; 71: 39-52.

6. Sharshar T, Gray F, Lorin de la Grandmaison G, Hopkinson NS, Ross E, Dorandeu A, et al. Apoptosis of neurons in cardiovascular autonomic centres triggered by inducible nitric oxide synthase after death from septic shock. Lancet 2003; 362: 1799-805.

7. Siami S, Annane D, Sharshar T. The encephalopathy in sepsis. Crit Care Clin 2008; 24: 67-82, viii.

8. Sonneville R, Verdonk F, Rauturier C, Klein IF, Wolff M, Annane D, et al. Understanding brain dysfunction in sepsis. Ann Intensive Care 2013; 3: 15 .
9. Iacobone E, Bailly-Salin J, Polito A, Friedman D, Stevens RD, Sharshar T. Sepsis-associated encephalopathy and its differential diagnosis. Crit Care Med 2009; 37: S331-6.

10. Young GB, Bolton CF, Austin TW, Archibald YM, Gonder J, Wells GA. The encephalopathy associated with septic illness. Clin Invest Med 1990; 13: 297-304.

11. Bolton CF, Young GB, Zochodne DW. The neurological complications of sepsis. Ann Neurol 1993; 33: 94-100.

12. Oddo M, Taccone FS. How to monitor the brain in septic patients? Minerva Anestesiol 2015; 81: 776-88.

13. O'Gorman RL, Poil SS, Brandeis D, Klaver P, Bollmann S, Ghisleni C, et al. Coupling between resting cerebral perfusion and EEG. Brain Topogr 2013; 26: 442-57.

14. Oddo M, Carrera E, Claassen J, Mayer SA, Hirsch LJ. Continuous electroencephalography in the medical intensive care unit. Crit Care Med 2009; 37: 2051-6.

15. Watson PL, Shintani AK, Tyson R, Pandharipande PP, Pun BT, Ely EW. Presence of electroencephalogram burst suppression in sedated, critically ill patients is associated with increased mortality. Crit Care Med 2008; 36: 3171-7.

16. Hosokawa K, Gaspard N, Su F, Oddo M, Vincent JL, Taccone FS. Clinical neurophysiological assessment of sepsis-associated brain dysfunction: a systematic review. Crit Care 2014; 18: 674.

17. Ziaja M. Septic encephalopathy. Curr Neurol Neurosci Rep 2013; 13: 383.

18. Foreman B, Claassen J, Abou KK, Jirsch J, Alschuler DM, Wittman J, et al. Generalized periodic discharges in the critically ill: a case-control study of 200 patients. Neurology 2012; 79: 1951-60.

How to cite this article: Velissaris D, Pantzaris ND, Skroumpelou A, Polychronopoulos $\mathrm{P}$, Karamouzos V, Pierrakos C, et al. Electroencephalographic abnormalities in sepsis patients in correlation to the calculated prognostic scores: A case series. J TransI Intern Med 2018; 6: 176-80. 